\title{
Accurate multi-robot targeting for keyhole neurosurgery based on external sensor monitoring
}

Proc IMechE Part H:

$\mathrm{J}$ Engineering in Medicine 226(5) 347-359

(c) IMechE 2012

Reprints and permissions:

sagepub.co.uk/journalsPermissions.nav DOI: $10.1177 / 0954411912442120$

pih.sagepub.com

(SAGE

\author{
Mirko Daniele Comparetti' , Alberto Vaccarella', Ilya Dyagilev², \\ Moshe Shoham², Giancarlo Ferrigno' and Elena De Momi ${ }^{1,3}$
}

\begin{abstract}
Robotics has recently been introduced in surgery to improve intervention accuracy, to reduce invasiveness and to allow new surgical procedures. In this framework, the ROBOCAST system is an optically surveyed multi-robot chain aimed at enhancing the accuracy of surgical probe insertion during keyhole neurosurgery procedures. The system encompasses three robots, connected as a multiple kinematic chain (serial and parallel), totalling I 3 degrees of freedom, and it is used to automatically align the probe onto a desired planned trajectory. The probe is then inserted in the brain, towards the planned target, by means of a haptic interface. This paper presents a new iterative targeting approach to be used in surgical robotic navigation, where the multi-robot chain is used to align the surgical probe to the planned pose, and an external sensor is used to decrease the alignment errors. The iterative targeting was tested in an operating room environment using a skull phantom, and the targets were selected on magnetic resonance images. The proposed targeting procedure allows about $0.3 \mathrm{~mm}$ to be obtained as the residual median Euclidean distance between the planned and the desired targets, thus satisfying the surgical accuracy requirements $(\mathrm{I} \mathrm{mm})$, due to the resolution of the diffused medical images. The performances proved to be independent of the robot optical sensor calibration accuracy.
\end{abstract}

\section{Keywords}

Robotic neurosurgery, visual servoing, iterative targeting

Date received: 6 December 20II; accepted: 23 February 2012

\section{Introduction}

During keyhole neurosurgical procedures, straight probes are introduced inside the brain. Suitable trajectories are planned on pre-operative medical images, e.g. for deep brain stimulation, StereoEEG and biopsies. ${ }^{1}$

Keyhole neurosurgery is a highly demanding surgical procedure, since functional areas of high importance have to be avoided during the probe advancement inside the brain. Robotic systems have already been used to automatically position and insert straight probes into the brain. ${ }^{2,3}$

Passive systems, such as as the Neuromate (Renishaw Ltd, UK), ${ }^{4}$ autonomously move to a predefined position (e.g. close to the entry point on the skull) before locking and powering off, then the probe is manually inserted by the surgeon. In the so-called 'semi-active' robotic systems, the surgeon can interact with the robot through a master handling device such as in the NeuroArm, which was developed for micro-surgery and stereotactic brain procedures. ${ }^{5}$ In semi-active systems, the probe can also be cooperatively driven (hands-on control) as in the ROSA ${ }^{\mathrm{TM}}$ (MedTech, France) system. ${ }^{6}$

When using a robot, the patient's medical images and the pre-operative plan must be registered with the robot reference frame in order to perform the planned probe targeting. This can be achieved using a laser pointer, ${ }^{7}$ fiducials placed on the patient's head ${ }^{8}$ or ultrasound localization systems. ${ }^{9,10}$ Optical tracking

\footnotetext{
'Bioengineering Department, Neuroengineering and Medical Robotics Laboratory, Politecnico di Milano, Italy

${ }^{2}$ Department of Mechanical Engineering, Technion - Israel Institute of Technology, Israel

${ }^{3}$ Istituto di Tecnologie Industriali ed Automazione, Consiglio Nazionale delle Ricerche, Italy
}

\section{Corresponding author:}

Mirko Daniele Comparetti, Bioengineering Department,

Neuroengineering and Medical Robotics Laboratory, Politecnico di

Milano, Piazza Leonardo da Vinci 32, Milan, Italy.

Email: mirko.comparetti@mail.polimi.it 
systems can also be used for guiding the surgical robotic arm (e.g. for total hip replacement, ${ }^{11}$ for neurosurgery ${ }^{12}$ and for bone ablation ${ }^{13}$ ). The integration of the robot and external localization systems requires a calibration procedure in order to establish a common reference frame. Hand-eye calibration allows mapping sensorcentred measurements into the robot/world frame. ${ }^{14}$

Optical tracking systems were also used to correct the pose of the robot when deviations between the actual and the planned position were detected, tracking the robot base and the tool relative to the patient. ${ }^{15-17}$ Cornellà et al. ${ }^{15}$ used tracking systems to map the position of the target to the robot reference frame, correcting the position of the robot, updating calibration parameters by means of a Kalman filter. Baron et al. ${ }^{17}$ implemented the control of the robot position and orientation using proportional integral (PI) controllers. Residual targeting errors are approximately twice as big as the localization errors.

Tracking system performance for robot control was also improved by adding inertial measurements units ${ }^{18}$ in order to increase the control robustness with respect to marker occlusions, to compensate for delay of the optical system and to reduce noise.

Serial robots generally have a large workspace, and their absolute positioning accuracy is low because of modelling errors in the kinematics. On the contrary, parallel robots have greater accuracy, but they suffer from a limited workspace relative to their footprint. Using robots connected in series, having a redundant number of degrees of freedom (DoFs), allows the performance of surgical tasks with a more flexible robot configuration in the operating room (OR) environment. A multi-robot approach for neurosurgery applications was already proposed in the Evolution I, ${ }^{19}$ designed for micro-neurosurgical and micro-endoscopic applications. A parallel kinematic machine (PKM) was coupled with an articulated mobile platform, achieving $20 \mu \mathrm{m}$ as the positioning accuracy. Despite the great targeting accuracy reached, the research was dismissed since the developed system was too cumbersome and expensive.

As in the aforementioned papers, the approach here presented combines navigation data via optical sensors and robotic assistance for keyhole neurosurgery using three robots connected as a multiple kinematic chain. The system is part of a robotic suite for keyhole neurosurgery, named ROBOCAST. A biopsy probe is positioned by the modular robotic system at the planned entry point on the patient skull, and is then inserted in the brain via tele-operation control through a haptic device. The previous work, i.e. the general ROBOCAST system architecture and pre-operative planning algorithms, was described by De Momi and Ferrigno $^{20}$ and Comparetti et al. ${ }^{21}$

In order to perform the targeting, an optical system is used to detect the position of the robots, of the patient and of the surgical tool held by the robot, allowing procedure navigation similar to that in
Cornellà et al. ${ }^{15}$ and Baron et al. ${ }^{17}$ In our approach, the external sensor allows iterative corrections of the pose of the robots in case errors between the desired and the actual poses of the surgical tool are detected. The correction movement is differently weighed in case the error (in terms of position and orientation error) is increasing or decreasing in magnitude.

The method proposed can be used with inexpensive and low-resolution manipulators, which would help reduce the cost of a medical robotic system, since the residual errors can be reduced by measurements and corrections; in order for the residual error to improve (i.e. get smaller), there should not be compliance in the kinematic chain (i.e. a stiff system is still needed) and placement resolution should still be high (i.e. small positional increments should be possible). These two requirements would limit how inexpensive the hardware can be made.

The main contribution of the paper is therefore to prove the clinical applicability of a navigated robotic approach for neurosurgical interventions. Only using the external localization system, the automatic robotic approach can satisfy the application accuracy requirements.

The original concept behind the iterative targeting algorithm was proposed by Comparetti et al. ${ }^{22}$ In the present paper, the suitability for clinical applicability has been proved through extensive tests, performed under operating condition settings, since the obtained targeting accuracy satisfies the clinical requirements. The innovative targeting algorithm presented has potential applications for surgical robotic systems already present in the market.

In addition, the control algorithm checks the overall system safety: whenever inconsistency is detected among redundant sensors (robot encoders versus optical tracking system), the system raises a warning and blocks the advancement of the probe inside the brain.

\section{Materials and methods}

\section{The system}

The ROBOCAST system is a robotic chain of three robots, totalling $13 \mathrm{DoFs}$, as explained below. As described by De Momi and Ferrigno, ${ }^{20}$ the system encompasses the following (see Table 1 for details):

- gross positioner (GP) (PathFinder, Prosurgics Ltd, UK), a serial 6 DoFs arm that is used to approach the patient head; ${ }^{23}$

- fine positioner (FP) (SpineAssist, Mazor, Israel), a parallel 6 DoFs miniaturized PKM, used to further correct the targeting, and which is rigidly connected to the GP via a custom-built quick-release interlock;

- linear actuator (LA), a 1 DoF bespoke piezoactuator that makes the biopsy linear probe advance through a tele-operated haptic interface 
Table I. Robot characteristics.

\begin{tabular}{llllll}
\hline Robot & Architecture & DoFs & Accuracy & Velocity & Workspace \\
\hline GP & Serial & 6 & $0.5 \mathrm{~mm}^{8}$ & $50 \mathrm{~mm} \mathrm{~s}^{-1^{*}}$ & $0.75 \mathrm{~m} \times 0.75 \mathrm{~m} \times 0.75 \mathrm{~m}^{*}$ \\
FP & Parallel & 6 & $<0.1 \mathrm{~mm}$ & $1.3 \mathrm{~mm} \mathrm{~s}^{-1}, 4.3^{\circ} \mathrm{s}^{-1}$ & $40 \mathrm{~mm} \times 40 \mathrm{~mm} \times 10 \mathrm{~mm}, 12^{\circ} \times 12^{\circ} \times 12^{\circ}$ \\
LA & Linear & 1 & $8 \mu \mathrm{m}$ & $2 \mathrm{~mm} \mathrm{~s}^{-1}$ & $110 \mathrm{~mm}$ \\
\hline
\end{tabular}

Fixed by ROBOCAST specifications.

(Omega, Force Dimension, Switzerland) ${ }^{24}$ the LA is attached to the FP end-effector upper plate.

The Certus optical tracking system (NDI, Ontario, Canada), with $0.15 \mathrm{~mm}$ stated accuracy, is used for tracking the overall robotic chain. Dynamic reference frames (DRFs), rigid bodies composed of four active markers each, are attached to all the bases and the end effectors of the robots (Table 2). The tracking system is interfaced with the robots high-level controller through a Sensor Manager application, ${ }^{25,26}$ which is based on the Image-guided Surgery Toolkit framework (Kitware Inc., Clifton Park, New York, USA, www.igstk.org).

The desired target in the brain and the entry point on the patient skull are defined on medical images during the planning. In the intra-operative scenario, the surgical tool is firstly aligned onto the desired trajectory at the entry point using the GP and the FP, and then inserted inside the brain towards the planned target, using the tele-operated LA.

\section{Spatial relations}

Calibrating the system means to establish a common reference frame among the robots (GP, FP and LA) and the optical sensor. To compute the spatial transformations (see Figure 1) among the forward kinematics of the robot, the joint angle encoders $\left(\mathbf{B}_{\mathrm{GP}}, \mathbf{B}_{\mathrm{FP}}\right.$ and $\left.\mathbf{B}_{\mathrm{LA}}\right)$ and the optical tracking system $\left(\mathbf{A}_{\mathrm{GP}}, \mathbf{A}_{\mathrm{FP}}\right.$ and $\mathbf{A}_{\mathrm{LA}}$ ), the approach described by Strobl and Hirzinger ${ }^{14}$ and De Momi et al. ${ }^{27}$ was used (GP and FP calibration). $\mathbf{X}_{\mathrm{GP}}, \mathbf{Y}_{\mathrm{GP}}, \mathbf{X}_{\mathrm{FP}}$ and $\mathbf{Y}_{\mathrm{FP}}$ are the transformations computed during the calibration and represent the transformations between the DRFs in the optical reference frame and the robots internal reference frame (see Figure 1):

Table 2. List of the DRFs of the ROBOCAST system and schematic representation (code). DRFs are visible in Figure I.

\begin{tabular}{lc}
\hline Position & Code \\
\hline GP base & B \\
GP end effector & E \\
FP base & E \\
FP end effector & $\perp$ \\
LA base & $\perp$ \\
LA end effector & $\dagger$ \\
\hline
\end{tabular}

- $\mathbf{X}_{\mathrm{GP}}$ is the transformation between the GP endeffector internal reference frame and the origin of the DRF attached to the GP end effector;

- $\mathbf{Y}_{\mathrm{GP}}$ is the transformation between the GP base internal reference frame and the origin of the DRF attached to the GP base;

- $\mathbf{X}_{\mathrm{FP}}$ is the transformation between the FP endeffector internal reference frame and the origin of the DRF attached to the FP end effector;

- $\mathbf{Y}_{\mathrm{FP}}$ is the transformation between the FP base internal reference frame and the origin of the DRF attached to the FP base;

- $\mathbf{X}_{\mathrm{LA}}$ is the transformation between the LA endeffector internal reference frame and the origin of the DRF attached to the LA end effector;

- $\mathbf{Y}_{\mathrm{LA}}$ is the transformation between the LA base internal reference frame and the origin of the DRF attached to the LA base.

In order to perform the calibration, the GP working volume was sampled, acquiring 28 poses (in a sphere of $173.21 \mathrm{~mm}$ radius). In order to sample the FP working volume as well, 28 poses were acquired (in a sphere of $7.86 \mathrm{~mm}$ in radius). Calibration errors were computed on 15 poses not used for calibration.

In order to calibrate the LA, the probe was moved for $100 \mathrm{~mm}$ (backwards and forwards with $5 \mathrm{~mm}$ spacing) and the three-dimensional (3D) coordinates of the tip position were sampled using a custom-built divot, equipped with a DRF. Principal component analysis ${ }^{28}$

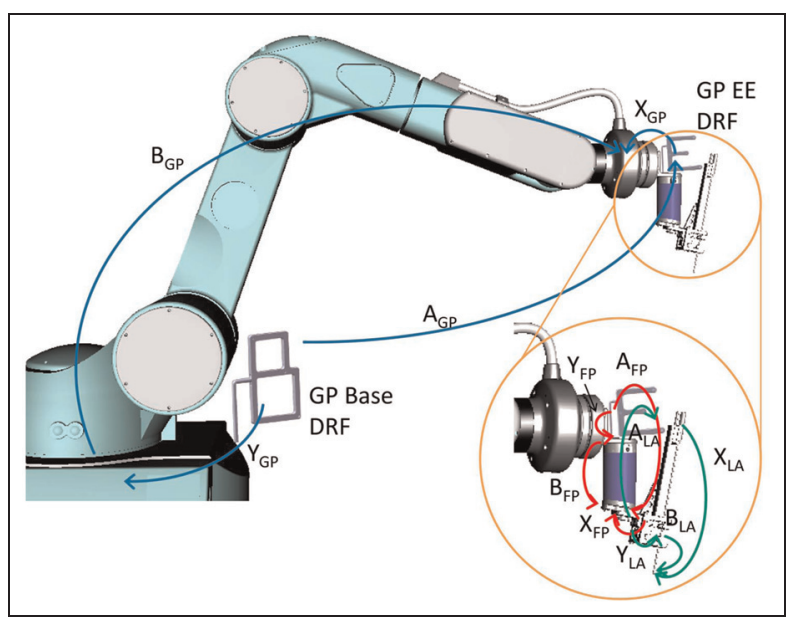

Figure I. Spatial transformations between the robot internal reference system and the DRF in Table 2. 
was applied in order to compute the orientation of the line approximating the probe advancement in the $3 \mathrm{D}$ space.

Probe targeting brings the probe (Tool) tip reference frame $\mathbf{T}_{j}$ in the desired target pose $\mathbf{T}_{d}(2 \mathrm{~cm}$ outside the patient skull entry point), as planned in the preoperative phase. From there, the probe is made in advance by the surgeon using the haptic interface. ${ }^{20}$ In order for the Tool $\mathbf{T}_{j}$ reference frame to reach the $\mathbf{T}_{d}$ reference frame, the $i$-th robot $(i=\mathrm{GP}, \mathrm{FP})$ has to change its pose from $\mathbf{B}_{i, j}$ to $\mathbf{B}_{i, j+1}$ (Figure 2), where $j$ is the movement iteration index.

If $\mathbf{M}_{i}$ represents the transformation between $\mathbf{T}_{j}$ and the $i$-th robot end-effector reference frame, the transformation to be applied to the $i$-th robot end effector $\mathbf{C}_{i, j}$ is

$$
\mathbf{C}_{i, j}=\mathbf{M}_{i} \cdot \mathbf{R}_{j} \cdot \mathbf{M}_{i}^{-1}
$$

where $\mathbf{R}_{j}$ is the transformation between the probe tip reference frame $\left(\mathbf{T}_{j}\right)$ and the target pose $\left(\mathbf{T}_{d}\right)$. Therefore, the new robot pose $\mathbf{B}_{i, j+1}$ is

$$
\mathbf{B}_{i, j+1}=\mathbf{B}_{i, j} \cdot \mathbf{C}_{i, j}
$$

Note that this transformation is independent of the base reference frame, and depends only on optical differential measurements $\left(\mathbf{R}_{j}\right)$, calibration matrices $\left(\mathbf{X}_{i}, \mathbf{Y}_{i}\right)$ and robot initial poses $\left(\mathbf{B}_{i, j}\right)$

$$
\mathbf{M}_{\mathrm{GP}}=\mathbf{X}_{\mathrm{GP}}^{-1} \cdot \mathbf{Y}_{\mathrm{FP}} \cdot \mathbf{B}_{\mathrm{FP}} \cdot \mathbf{X}_{\mathrm{FP}}^{-1} \cdot \mathbf{Y}_{\mathrm{LA}} \cdot \mathbf{B}_{\mathrm{LA}}
$$

for the GP robot $(i=\mathrm{GP})$ and

$$
\mathbf{M}_{\mathrm{FP}}=\mathbf{X}_{\mathrm{FP}}^{-1} \cdot \mathbf{Y}_{\mathrm{LA}} \cdot \mathbf{B}_{\mathrm{LA}}
$$

for the FP robot $(i=\mathrm{FP})$.

\section{The closed-loop targeting algorithm}

The residual transformation $\mathbf{R}_{j}$ between the desired $\left(\mathbf{T}_{d}\right)$ and the actual $\left(\mathbf{T}_{j}\right)$ probe tip reference frame is

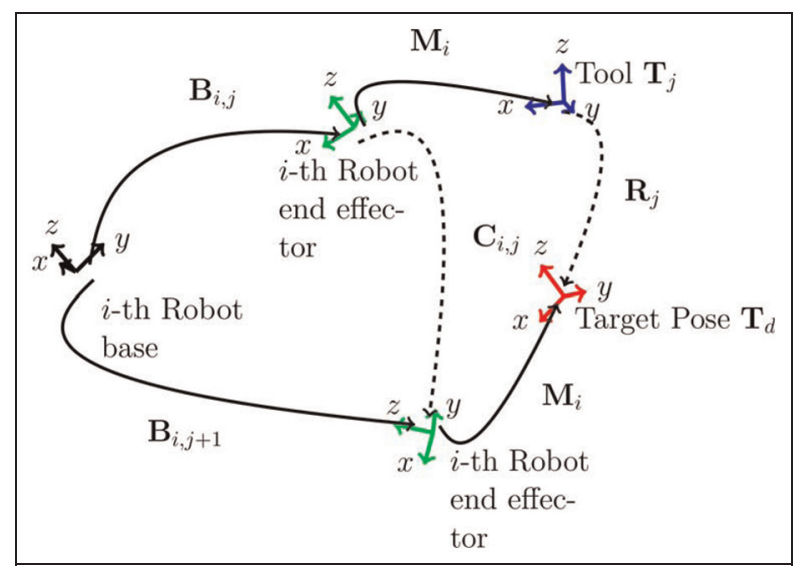

Figure 2. Spatial transformations used during probe targeting. $\mathbf{M}_{i}$ is the spatial transformation between the Tool and the robot end effector: for the GP robot, it is $\mathbf{M}_{\mathrm{GP}}=\mathbf{X}_{\mathrm{GP}}^{-1} \cdot \mathbf{Y}_{\mathrm{FP}} \cdot \mathbf{B}_{\mathrm{FP}} \cdot \mathbf{X}_{\mathrm{FP}}^{-1}$. $\mathbf{Y}_{\mathrm{LA}} \cdot \mathbf{B}_{\mathrm{LA}}$, and for the FP robot, $\mathbf{M}_{\mathrm{FP}}=\mathbf{X}_{\mathrm{FP}}^{-1} \cdot \mathbf{Y}_{\mathrm{LA}} \cdot \mathbf{B}_{\mathrm{LA}}$. computed as $\mathbf{R}_{j}=\mathbf{T}_{j}^{-1} \cdot \mathbf{T}_{d}$. The translation error (translation component (TC)) is the Euclidean distance of the translation components of $\mathbf{R}_{j},{ }^{29}$ while the rotation error (rotation component (RC)) is estimated by computing the arctangent of the norm of the vector component of the quaternion of $\mathbf{R}_{j}$, divided by the scalar component.

The targeting algorithm is described in Figure 3: first, the $i$-th robot approaches the target with an iterative approach until the error $\mathbf{R}_{j}$ (both the translation (TC) and the rotation (RC) components) is below a threshold (specifically defined for each robot) or if the maximum number of iterations is reached. Then the $(i+1)$-th robot is moved and the control loop continues until success or failure are met. Note that, if $\mathbf{M}_{i}$ was known perfectly, just one movement would put the end effector in the correct position. Iteration is required to deal with $\mathbf{M}_{i}, \mathbf{B}_{i, j}$ and optical tracker inaccuracies.

The robot arm pose correction $\mathbf{C}_{i, j}$ (dashed block represented in Figure 3) is computed to reduce the $\mathbf{R}_{j}$ transformation in magnitude (in both TC and RC components), avoiding instability. The $\mathbf{R}_{j}$ translation

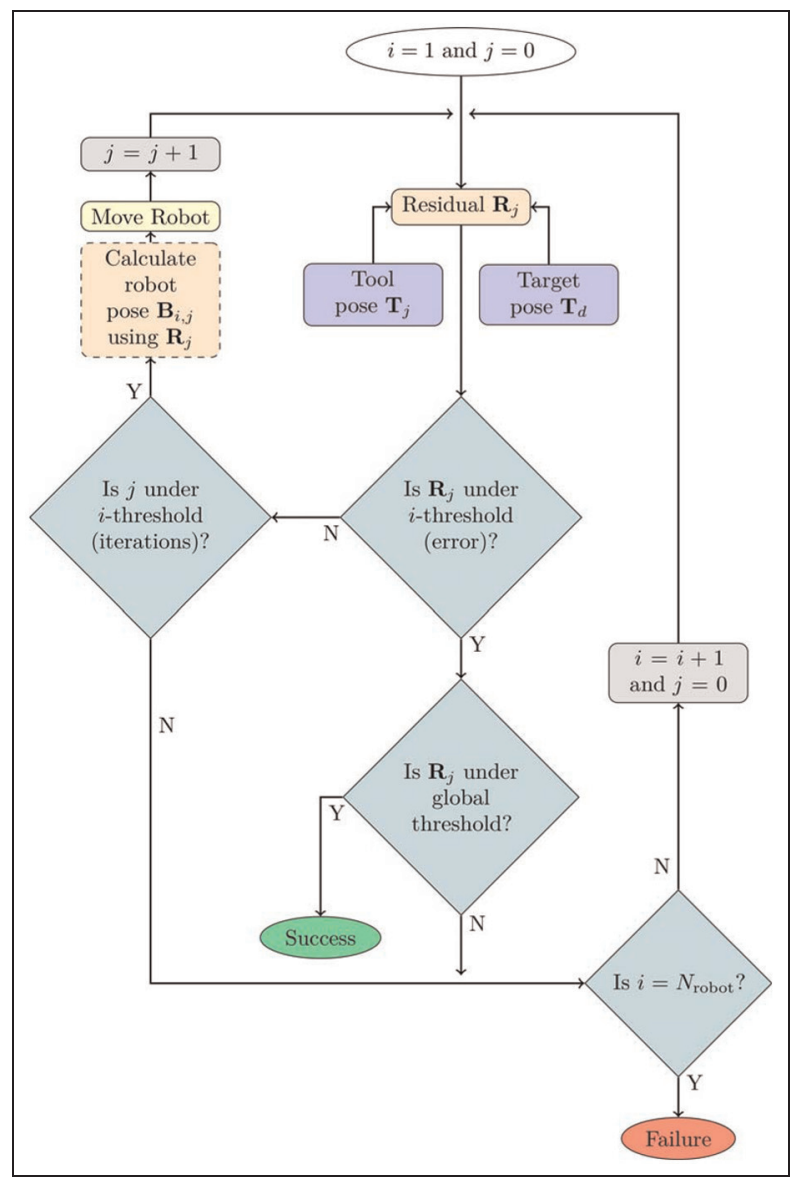

Figure 3. The residual transformation $\mathbf{R}_{j}$ is $\mathbf{R}_{j}=\mathbf{T}_{j}^{-1} \cdot \mathbf{T}_{d}$. First, the $i$-th robot approaches the target with an iterative approach until the error $\mathbf{R}_{j}$ (both the translation (TC) and the rotation (RC) components) is below a threshold (specifically defined for each robot) or if the maximum number of iterations is reached. Then, the $(i+1)$-th robot is moved, and the control loop continues until success or failure are met. 
component $\left(\mathrm{TC}_{j}\right)$ and the $\mathbf{R}_{j}$ rotation component $\left(\mathrm{RC}_{j}\right)$ were scaled by multiplying $\mathrm{TC}_{j}$ and $\mathrm{RC}_{j}$ times a value $n_{j}^{s}$, computed as follows

$$
n_{j}^{s}\left(\dot{x}_{j}^{s}\right)=\frac{1}{1+e^{\frac{\dot{x}_{j}^{s}}{10}}}
$$

where $\dot{x}$ is the first derivative, computed using finite differences, of the $s$ component $\left(\mathrm{TC}_{j}\right.$ and $\left.\mathrm{RC}_{j}\right)$. As an example, the correction $n$ is 0.5 when the error does not change from the previous iteration $\left(\mathrm{TC}_{j+1}=\mathrm{TC}_{j}\right.$ or $\mathrm{RC}_{j+1}=\mathrm{RC}_{j}$ ). When the error is decreasing, thus $\dot{x}<0$, the correction $n$ approaches 1 , while if the error increases, the correction $n$ is reduced towards 0 . The exponential parameter $1 / 10$, the sigmoid amplitude, was empirically set.

The error reduction algorithm is designed to allow the robot to get closer to the target, assuring convergence with a small iterations number.

\section{Experimental protocol}

The planning. A brain phantom was designed and developed using a plastic skull and polyvinyl alcohol as brain mimicking material. ${ }^{30,31}$ Two gadolinium markers (targets) were fixed on the base of the skull (Figure 4).
Magnetic resonance image (MRI) images of the phantom were acquired $(\mathrm{T} 1,512 \times 512 \times 144,0.5 \mathrm{~mm} \times$ $0.5 \mathrm{~mm} \times 1 \mathrm{~mm}$ slice spacing) and the $3 \mathrm{D}$ coordinates of the targets computed in the medical images reference frame. For each target point, 12 possible entry points were selected on the whole skull surface. Therefore, 12 probe trajectories were planned for each target point.

The planned trajectories were registered into the operating volume using a point-based registration algorithm. ${ }^{32}$

The targeting tests. The modular robotic system was moved in order to align the actual surgical probe trajectory $\left(\mathbf{T}_{j}\right)$ with the planned trajectory $\left(\mathbf{T}_{d}\right)$. The surgical probe was positioned by the GP and the FP $2 \mathrm{~cm}$ above the entry point along the planned trajectory $\left(\mathbf{T}_{d}\right)$, then it was inserted in the phantom by the LA towards the entry point and the target point, following the designed surgical plan. The GP threshold was set to $2 \mathrm{~mm}$ for translation (TC) and $0.1 \mathrm{rad}$ for rotation (RC), with a maximum of 10 iterations allowed, after which the FP was moved towards the desired target pose $\left(\mathbf{T}_{d}\right)$. The FP threshold was set to $0.08 \mathrm{~mm}$ (corresponding to the expected accuracy of DRFs ${ }^{33}$ for translation and 0.01 rad for rotation with a maximum of 10 iterations allowed).

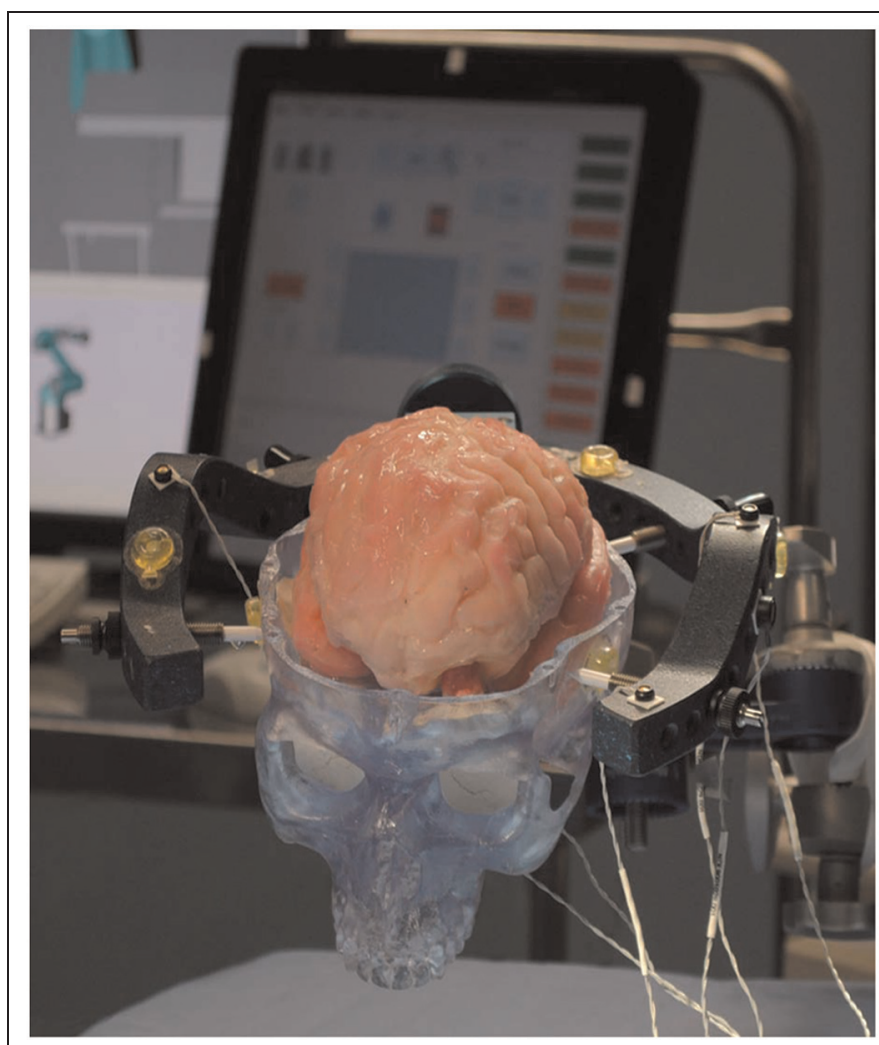

(a)

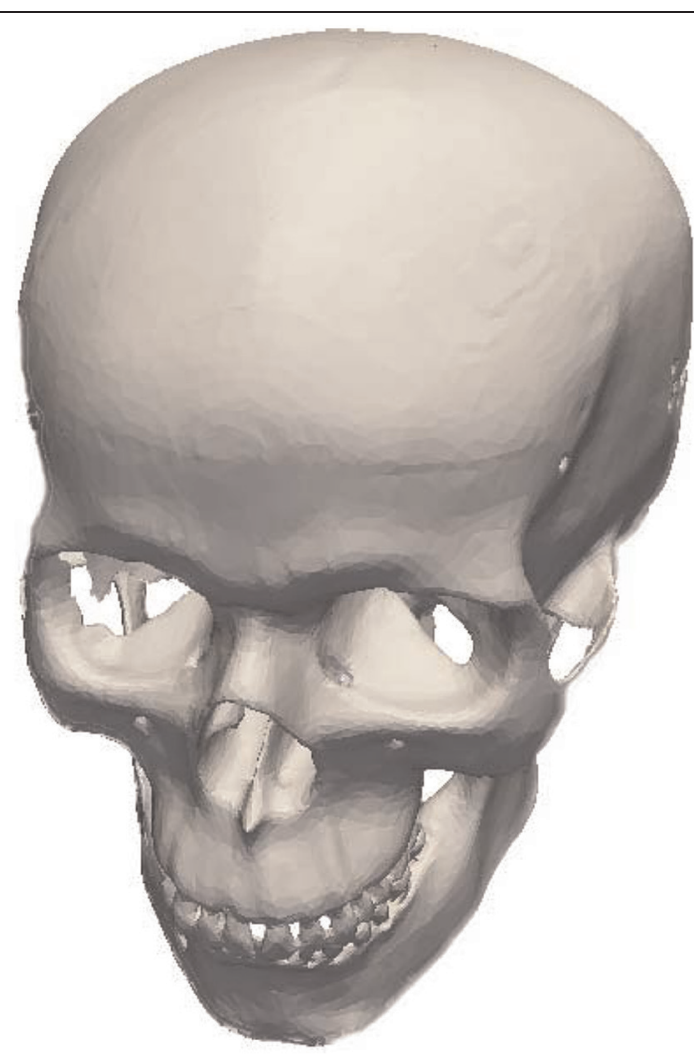

(b)

Figure 4. Brain phantom. (a) Skull and brain phantom with gadolinium markers and optical active markers attached on the magnetic resonance image (MRI) compatible head ring. (b) Skull model reconstructed from the MRI dataset. 
Targeting tests were performed with the ROBOCAST system in three different conditions.

1. 'Calibrated' targeting: the optical calibration of GP, FP and LA (described in the section on spatial relations) was performed immediately before the targeting experiments.

2. 'Non-calibrated' targeting: the optical calibration of GP, FP and LA was performed the day before the experiments, in order to check the calibration stability.

3. 'Perturbed' targeting. In order to test the algorithm robustness, the calibration (see the section on spatial relations) was altered on purpose by multiplying the calibration matrix $\left(\mathbf{X}_{\mathrm{GP}}\right)$ by transformation matrices $\left(\mathbf{P}_{\mathrm{GP}}\right)$, randomly chosen in a population of $0 \mathrm{~mm} . .50 \mathrm{~mm}$ translation and 0 rad..0.09 rad rotation (uniform distribution) and multiplying the calibration matrix $\left(\mathbf{X}_{\mathrm{FP}}\right)$ by transformation matrices $\left(\mathbf{P}_{\mathrm{FP}}\right)$ randomly chosen in a population of $0 \mathrm{~mm} . .5 \mathrm{~mm}$ range of translation and $0 \mathrm{rad} . .0 .03 \mathrm{rad}$ of rotation (uniform distribution).

For each targeting experiment (Figure 5), all DRFs poses were acquired at $30 \mathrm{~Hz}$.

The test protocol is reported in Table 3 .

The evaluation metrics and data analysis. In order to evaluate the system performances, the following data were measured:

- the final probe tip residual transformation matrices $\left(\mathbf{R}_{f}\right)$ components $\left(\mathrm{TC}_{\mathrm{f}}\right.$ and $\left.\mathrm{RC}_{\mathrm{f}}\right)$ for both the $\mathrm{GP}$ and the FP;
- the actual entry point (EP);

- the actual target point (TP);

- the actual probe trajectory (PT), computed as the line crossing EP and TP.

Targeting performance evaluation was performed computing the residual translation and rotation transformation $\left(\mathrm{TC}_{j}\right.$ and $\mathrm{RC}_{j}$ components of $\mathbf{R}_{j}$ ) for each targeting trial.

Experimental results were evaluated using a nonparametric Kruskal-Wallis test with $p<0.05$ significance (STATISTICA 10, StatSoft).

In order to check whether the iterative targeting always ensures that the residual translation transformation ( $\mathrm{TC}_{j}$ component of $\mathbf{R}_{j}$ ) is decreasing in magnitude, the following data were computed:

- iterative corrections of the $\mathrm{GP}\left(\mathrm{IC}_{\mathrm{GP}}\right)$, computed for each targeting experiment $(k)$ as

$$
\mathrm{IC}_{\mathrm{GP} k}=\mathrm{TC}_{\mathrm{GP}, j-1, k}-\mathrm{TC}_{\mathrm{GP}, j, k}
$$

- iterative correction of the $\mathrm{FP}\left(\mathrm{IC}_{\mathrm{FP}}\right)$, computed for each targeting experiment $(k)$ as

$$
\mathrm{IC}_{\mathrm{FP} k}=\mathrm{TC}_{\mathrm{FP}, j-1, k}-\mathrm{TC}_{\mathrm{FP}, j, k}
$$

The Pearson correlation coefficients among the TC component of the $\mathbf{P}_{\mathrm{GP}}$ and the TC component of the residual $\mathbf{R}_{j}$ and among the TC component of the $\mathbf{P}_{\mathrm{FP}}$ and the TC component of the residual $\mathbf{R}_{j}$ were computed.

In order to compare the overall targeting accuracy, the following evaluation metrics were computed:

- residual error at the entry point $\left(\mathrm{RE}^{\mathrm{EP}}\right)$ : Euclidean distance between the actual and the desired EP;

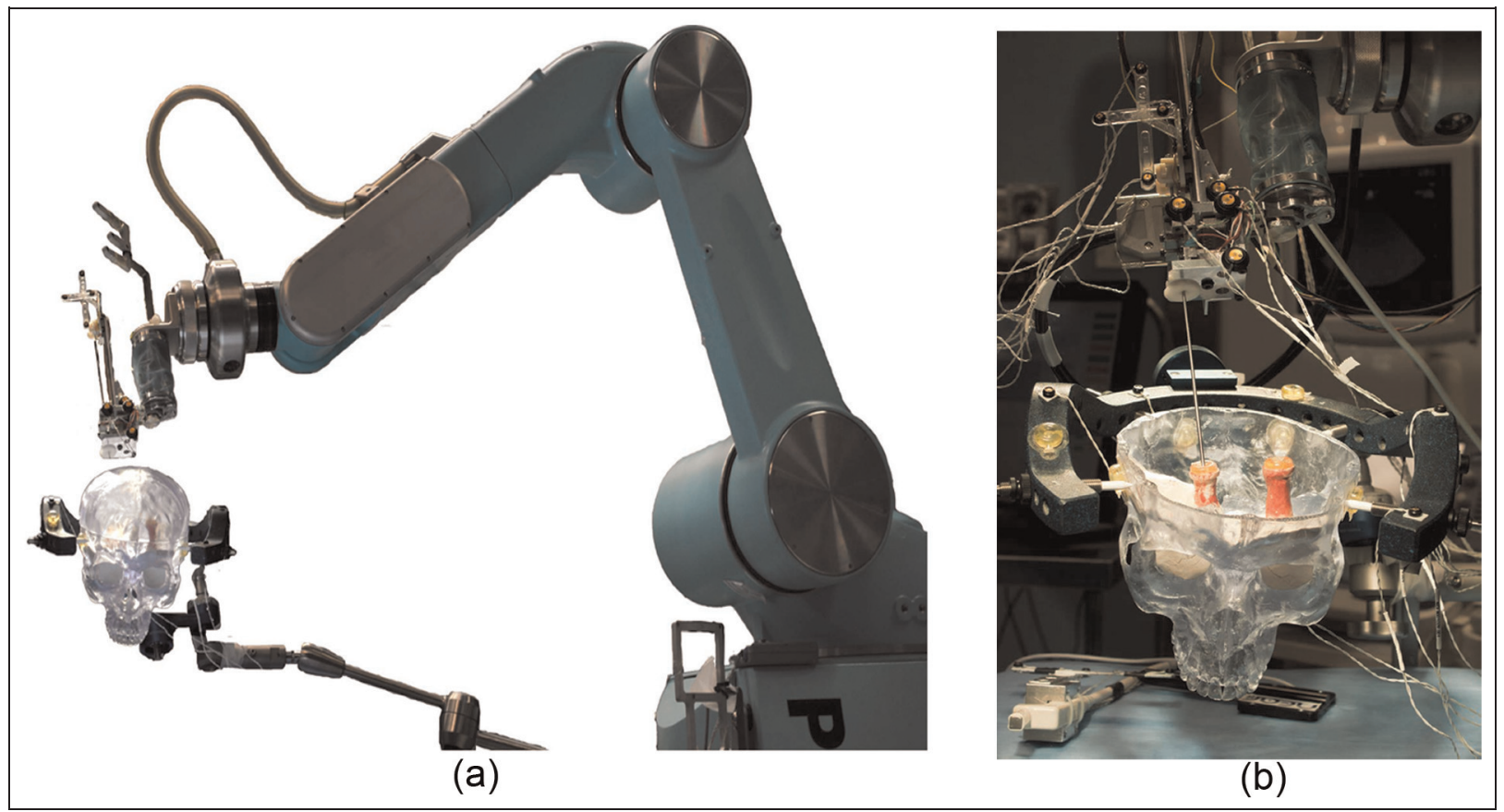

Figure 5. The OR set-up during experiments. (a) The surgical probe is automatically positioned by the GP and FP. (b) Final targeting verification. The probe tip reaches the gadolinium marker centre. 
Table 3. Experimental protocol.

\begin{tabular}{lll}
\hline $\begin{array}{l}\text { Number of targets } \\
\text { Number of entry points } \\
\text { (for each target) }\end{array}$ & 2 & \\
$\begin{array}{l}\text { Number of repetitions in } \\
\text { each entry point }\end{array}$ & 3 & \\
$\begin{array}{l}\text { Maximum number of iteration } \\
\text { per robots }\end{array}$ & 10 & \\
& $\begin{array}{l}\text { Translation } \\
(\mathrm{mm})\end{array}$ & Angle (rad) \\
GP threshold & 2 & 0.1 \\
FP threshold & 0.08 & 0.01 \\
GP calibration perturbation $\left(\mathbf{P}_{\mathrm{GP}}\right)$ & 50 (max.) & 0.09 (max.) \\
FP calibration perturbation $\left(\mathbf{P}_{\mathrm{FP}}\right)$ & 5 (max.) & 0.03 (max.) \\
\hline
\end{tabular}

- residual error at the target point $\left(\mathrm{RE}^{\mathrm{TP}}\right)$ : Euclidean distance between the actual and the desired TP;

- angle between the actual and desired probes trajectories $\left(\mathrm{A}^{\mathrm{PT}}\right)$ : angle between the two lines in space.

Different experimental setups were compared using a non-parametric Kruskal-Wallis test with $p<0.05$ significance (STATISTICA 10, StatSoft).

\section{Results}

\section{Calibration}

Calibration residuals and calibration errors for GP, FP and LA are reported in Table 4 in both the translation (TC) and the rotation (RC) components. Calibration residuals are about $0.2 \mathrm{~mm}$, which is the accuracy that would have been reached without an iterative targeting, if the calibration model would perfectly generalize.

GP and FP targeting. Figure 6 shows the $\mathrm{TC}_{\mathrm{F}}$ and $\mathrm{RC}_{\mathrm{F}}$ components of the final (either below threshold or after the maximum number of iterations was reached) $\mathbf{R}_{j}$ transformation matrix for the GP and the FP in the three evaluation scenarios (calibrated, non-calibrated and perturbed). There is no significant difference within

Table 4. Calibration residuals and calibration errors (median, 25 th and 75 th percentile) for the three robots (GP, FP and LA).

\section{Calibration residual}

\begin{tabular}{lll}
\hline & TC $(\mathrm{mm})$ & $\mathrm{RC}(\mathrm{mm})$ \\
\hline GP & $0.2245(0.1288 . .0 .2623)$ & $0.0021(0.0008 . .0 .0306)$ \\
FP & $0.1548(0.1260 . .0 .2009)$ & $0.0035(0.0020 .0 .0049)$ \\
LA & $0.2561(0.1452 . .0 .4279)$ & $0.0014(0.0009 . .0 .0054)$ \\
\hline
\end{tabular}

Calibration errors

\begin{tabular}{lll}
\hline & TC $(\mathrm{mm})$ & $\mathrm{RC}(\mathrm{rad})$ \\
\hline GP & $0.7724(0.6430 . .0 .8303)$ & $0.0008(0.0006 .00 .001 \mathrm{I})$ \\
FP & $0.2405(0.2025 .0 .3450)$ & $0.0129(0.0115 .0 .0140)$ \\
LA & $0.2863(0.2639 .0 .34 \mathrm{II})$ & $0.0073(0.0069 .0 .0077)$
\end{tabular}

the same robot (GP or FP) in the three evaluation scenarios as far as the $\mathrm{TC}_{\mathrm{F}}$ parameter is considered. The angular residual error of the GP $\left(\mathrm{RC}_{\mathrm{f}}\right)$ worsen when the system is not calibrated, while the $\mathrm{RC}_{\mathrm{f}}$ of the FP is not statistically affected. The FP provides a significantly better final pose of the surgical probe tip with respect to the GP robot.

Figure 7 shows $\mathrm{IC}_{\mathrm{GP}}$ (equation (6)) and $\mathrm{IC}_{\mathrm{FP}}$ (equation (7)) in the three evaluation scenarios (calibrated, non-calibrated and perturbed). The correction value population appears normally distributed, with few negative outliers (Table 5).

Figure 8 shows the relationship between the TC component of the $\mathbf{P}_{\mathrm{GP}}$ and the TC component of the $\mathbf{R}_{j}$, after the GP targeting movement, and between the TC component of the $\mathbf{P}_{\mathrm{FP}}$ and the TC component of the $\mathbf{R}_{j}$, after the FP targeting movement. As shown, there is no correlation $(p>0.05)$ among the perturbation and the automatic targeting performance, neither for the GP (Figure 8(a)), nor for the FP (Figure 8(b)).

\section{Linear actuator targeting}

Figure 9 shows the targeting distances at the entry point $\left(\mathrm{RE}^{\mathrm{EP}}\right)$, at the target point $\left(\mathrm{RE}^{\mathrm{TP}}\right)$ and the angular error among the planned and the actual probe trajectory $\left(\mathrm{A}^{\mathrm{PT}}\right)$ in the three evaluation scenarios (calibrated, non-calibrated and perturbed). As shown, there is a significant difference between the targeting errors TC at the EP and at the TP, and among the three calibration scenarios. In addition, there is a significant difference in the angular trajectory RC errors among the three calibration scenarios.

The location of the entry point did not influence the targeting accuracy, since no statistically significant difference was found.

\section{Discussion}

In this paper, we show the experimental evaluation of a novel targeting algorithm designed for a multi-robot system (three robots connected in series) designed for keyhole neurosurgery, based on external sensors. In neurosurgery, the desired robotic pose for automatic targeting is usually defined using a laser pointer or fluoroscopic images of the patient. ${ }^{9,34}$ Robotic systems suffer from two main drawbacks: the movement accuracy of a serial robot does not respect surgical requirements and there is a lack of feedback information if the actual robot pose differs from the planned desired one.

With regard to targeting performances, it is worth recalling that the ROBOCAST system was specially designed for keyhole approaches, where a straight surgical probe is inserted in the brain tissue and the robot acts as an assistant, providing an accurate holder for guiding the probe insertion. Therefore, the aim of this study was to improve and test the accuracy of targeting in terms of position and rotation errors. The novelty of the approach relies on the iterative tracking of the 


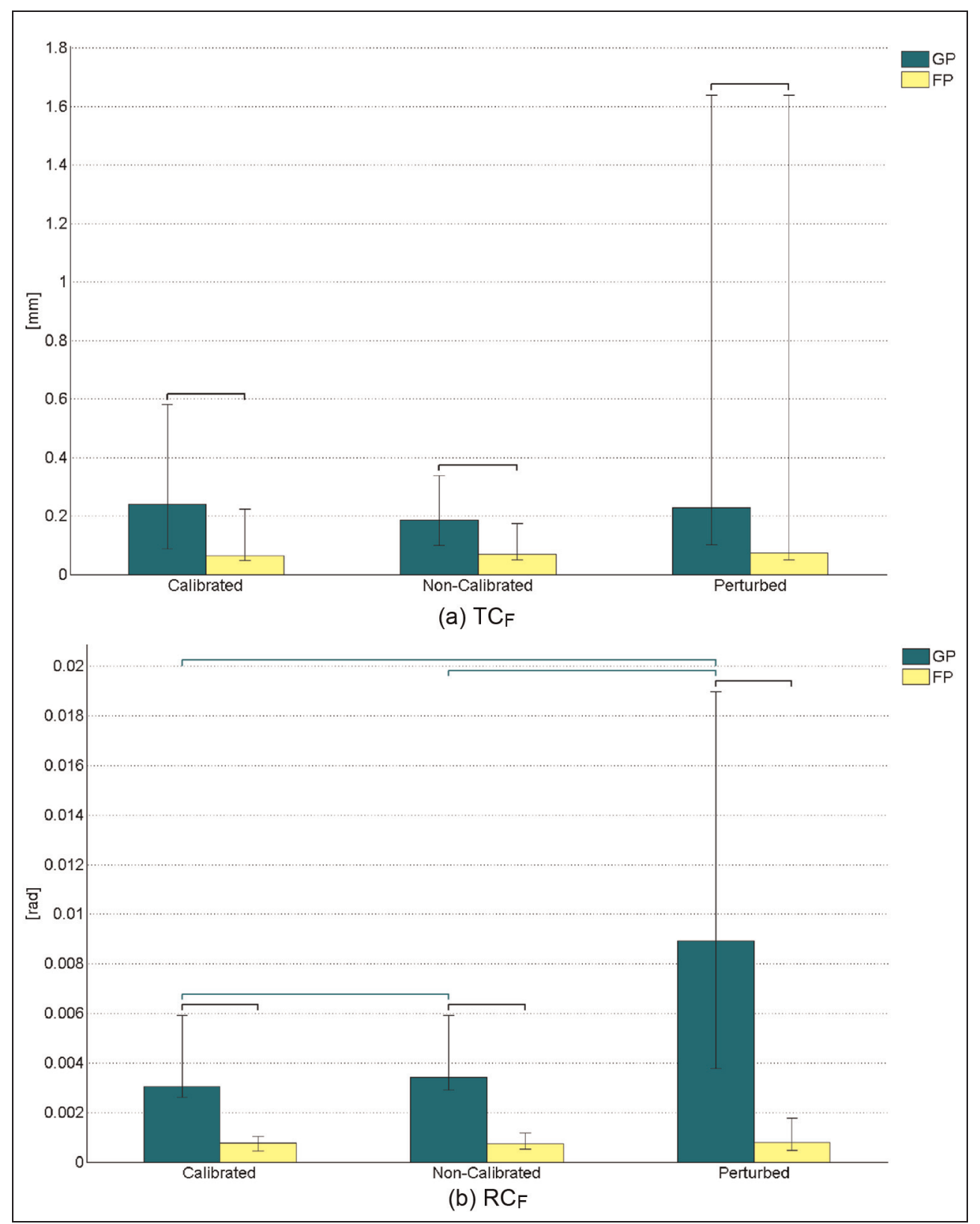

Figure 6. Components of the final (either below threshold or after the maximum number of iterations was reached) $\mathbf{R}_{j}$ transformation matrix for the GP and the FP in the three evaluation scenarios (calibrated, non-calibrated and perturbed). Bars represent the median value and the error bars show the interquartile range of the data presented. Horizontal bars indicate significant difference $(p<0.05)$.

current pose, performed combining the robotic architecture with an external optical tracking device. The control of the position and orientation is implemented by applying a correction factor that is scaled depending on the fact that the robot is getting closer or further to the target pose.

In the proposed approach, an optical localization system tracks both the pose of the patient head and the robot end effector, ${ }^{18}$ estimating the error between the planned and the current surgical tool pose, allowing further iterative corrections of the pose of the robot end effector. Also, kinematic redundancy (13 DoFs) allows the optimization of the robot approach: the miniaturized parallel robot (FP) mounted on the serial robot (GP) provides a more accurate surgical probe pose, due to its greater accuracy and resolution. The iterative targeting algorithm, which moves the second robot only after the GP residual error transformation has reached the pre-defined threshold, assures that the FP is able to perform further corrections within its limited workspace. The LA is then used to make the probe advance along the trajectory reached by the GP and FP. In order to apply such a hybrid configuration to the operating room, a smaller and more compact system has to be designed in the future (i.e. a smaller GP), but results reported still hold. 


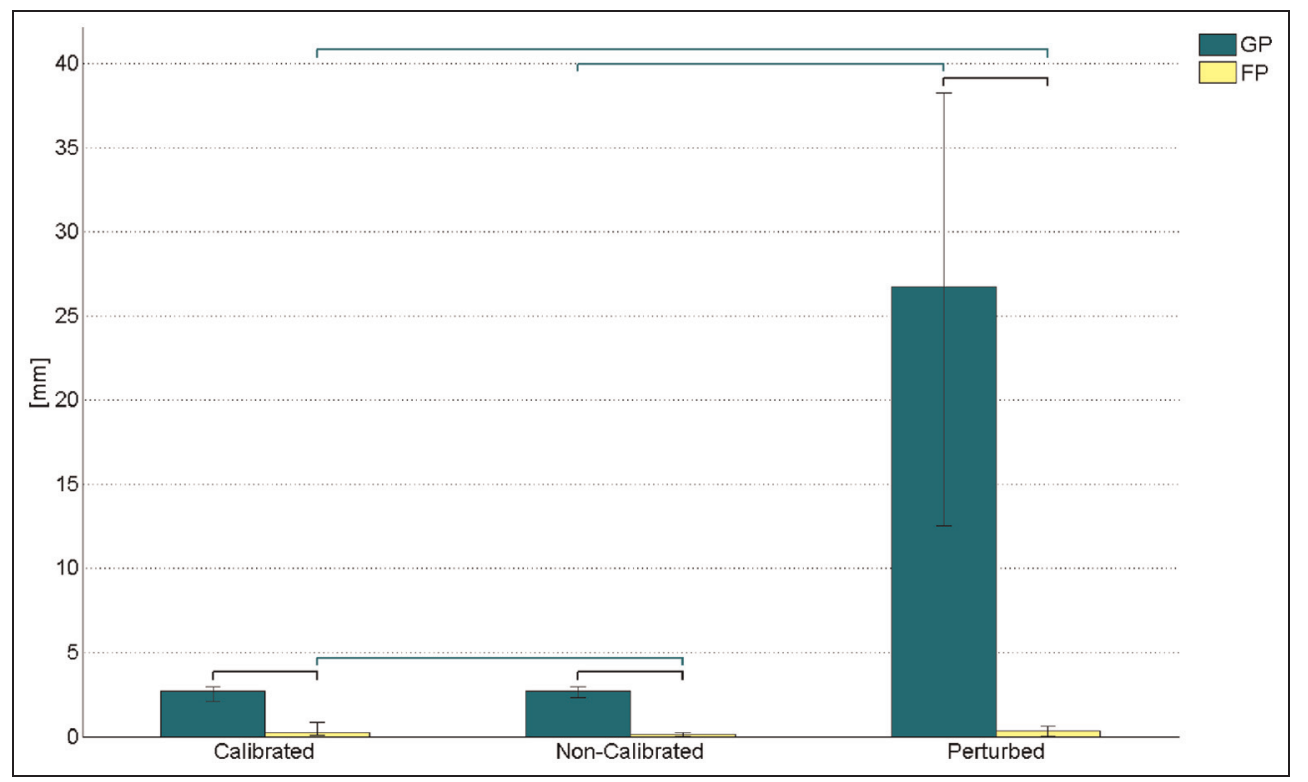

Figure 7. $I C_{\mathrm{GP}}$ (equation (6)) and $\mathrm{IC}_{\mathrm{FP}}$ (equation (7)) in the three evaluation scenarios (calibrated, non-calibrated and perturbed). Bars represent the median value and the error bars show the interquartile range of the data presented. Horizontal bars indicate significant difference $(p<0.05)$.

Table 5. Negative corrections of iterative algorithm of GP and FP targeting.

\begin{tabular}{llll}
\hline Condition & Value & GP & FP \\
\hline Calibrated & \# (\%) & I (0.49\%) & $6(3 \%)$ \\
& Largest & -0.1479 & -0.2066 \\
Non-calibrated & $\begin{array}{l}\text { correction (mm) } \\
\text { Perturbed }\end{array}$ & $0(0 \%)$ & $10(4.9 \%)$ \\
& $\begin{array}{l}\text { Largest } \\
\text { correction }(\mathrm{mm})\end{array}$ & 0 & $-0.401 \mathrm{I}$ \\
& \# (\%) & $0(0 \%)$ & $14(0 \%)$ \\
& Largest & 0 & -0.8775 \\
& correction $(\mathrm{mm})$ & & \\
\hline
\end{tabular}

The system was tested following the planned operatory workflow: first the GP and FP bring the probe's actual pose to the planned one, $2 \mathrm{~cm}$ above the entry point (GP, FP targeting). The residual error, detected as the difference between the current pose of the tip of the surgical probe with respect to the desired one in the optical reference system, is reduced iteratively, first approaching the reduction with the GP, then refining the targeting with the (FP).

The following calibration procedure was performed:

- right before the targeting;

- the day before the targeting;

- right before the targeting, then perturbed adding noise.

The targeting approach outperforms other neurosurgical robots targeting accuracies. As examples, the PathFinder was tested on phantoms and the reported targeting accuracy was $0.5 \mathrm{~mm} .^{8}$ The NeuroMate accuracy was reported to be $1.95 \mathrm{~mm}$ in the frameless approach $^{9}$ and $2.9 \mathrm{~mm}$ using implanted skull fiducials. ${ }^{10}$

In the literature, robotic targeting for keyhole surgery was already addressed providing optical feedback to robots. With respect to existing methodologies, the one proposed is based on iterative corrections and allows the reduction of targeting errors of about $50 \%$.

The optical feedback approach proposed by Cornellà et al. ${ }^{16}$ allowed $(2.6 \pm 0.8) \mathrm{mm}$ to be attained as the maximum error at the first iteration and $(0.60 \pm 0.36) \mathrm{mm}$ as the final targeting error, while Baron et al. ${ }^{17}$ attained $0.5 \mathrm{~mm}$ of accuracy without previous calibration. Using the iterative approach proposed, the GP allowed $0.25 \mathrm{~mm}$ to be attained as the median value in all three calibration scenarios, while the FP targeting performances were around $0.1 \mathrm{~mm}$ as the median value. It must be noted that this value is limited only by the registration error and by the accuracy of the localization system.

The iterative approach proposed proved to be independent from the calibration residuals: even if the calibration transformation is inaccurate, the iterative algorithm allows comparable residual errors to be attained, with accurate calibrations for both the serial and the parallel robots, despite the high initial errors.

The feedback control scheme is based on kinematics and it is intrinsically stable because of the low speed allowed. Stability problems were tackled by modulating the amplitude of the correction as a function of current error. In addition, the target was considered fixed since the patient head is supposed to be framed in a head ring.

The correction values of the GP are negative in magnitude (about $-0.2 \mathrm{~mm}$, which means that the robot is 


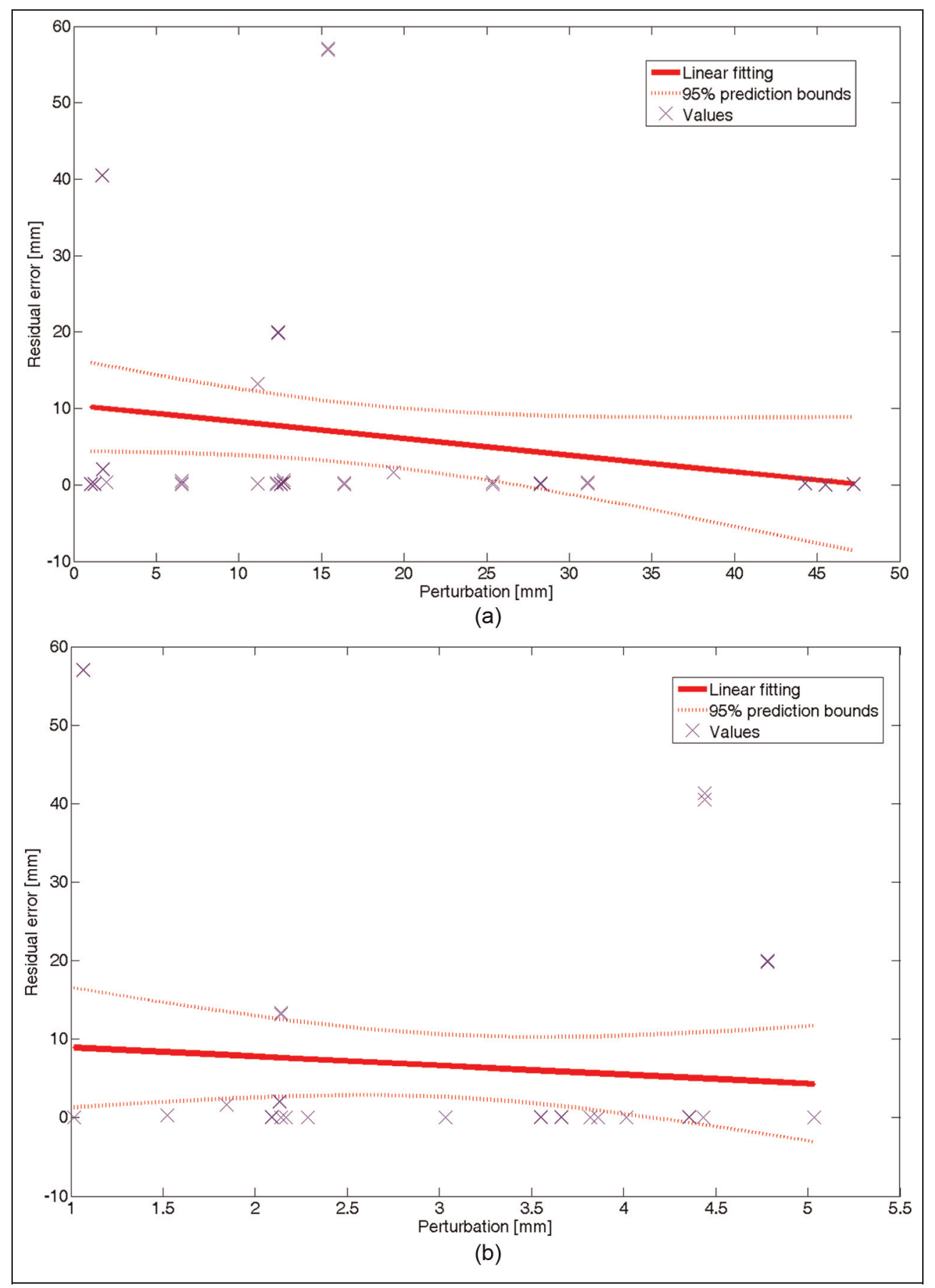

Figure 8. Relationship between the TC component of the $\mathbf{P}_{i-\text { th robot }}$ and the TC component of the $\mathbf{R}_{j}$ after the $i$-th robot movement; Pearson coefficient value and $p$ value are also reported. (a) Relationship between the TC component of the $\mathbf{P}_{\mathrm{GP}}$ (perturbation) and the TC component of the $\mathbf{R}_{j}$ (residual error) after GP movement; Pearson $-0.2 \mathrm{I} 2 \mathrm{I}, p=0$. I038. (b) Relationship between the TC component of the $\mathbf{P}_{\mathrm{FP}}$ (perturbation) and the TC component of the $\mathbf{R}_{j}$ (residual error) after FP movement; Pearson $-0.0948, p=0.4712$.

getting further from the target) in one case only when the system is calibrated. The correction values of the FP are negative in magnitude (about $-0.3 \mathrm{~mm}$ ) in approximately $5 \%$ of the cases, independently of the calibration accuracy. Such values are comparable to the optical tracking system accuracy. Stated optical system accuracy is $0.15 \mathrm{~mm}$, which is the worse $3 \mathrm{D}$ localization error. When using a four marker DRF, the target error in the centre of mass is $0.08 \mathrm{~mm} .{ }^{33}$ The external sensor accuracy (tracker) indeed affects the results, since the corrections are computed using the tracker coordinates. Non-expensive systems with low working volume, such as the Polaris Vicra system (NDI, Ontario, Canada), can be used for the target surgical scenario, which is surgery around the head, allowing $0.13 \mathrm{~mm}$ of accuracy to be attained in the case when four markers are used. ${ }^{33}$

Considering the LA targeting, the error at the entry point $(0.6 \mathrm{~mm}$ as median value) is statistically 


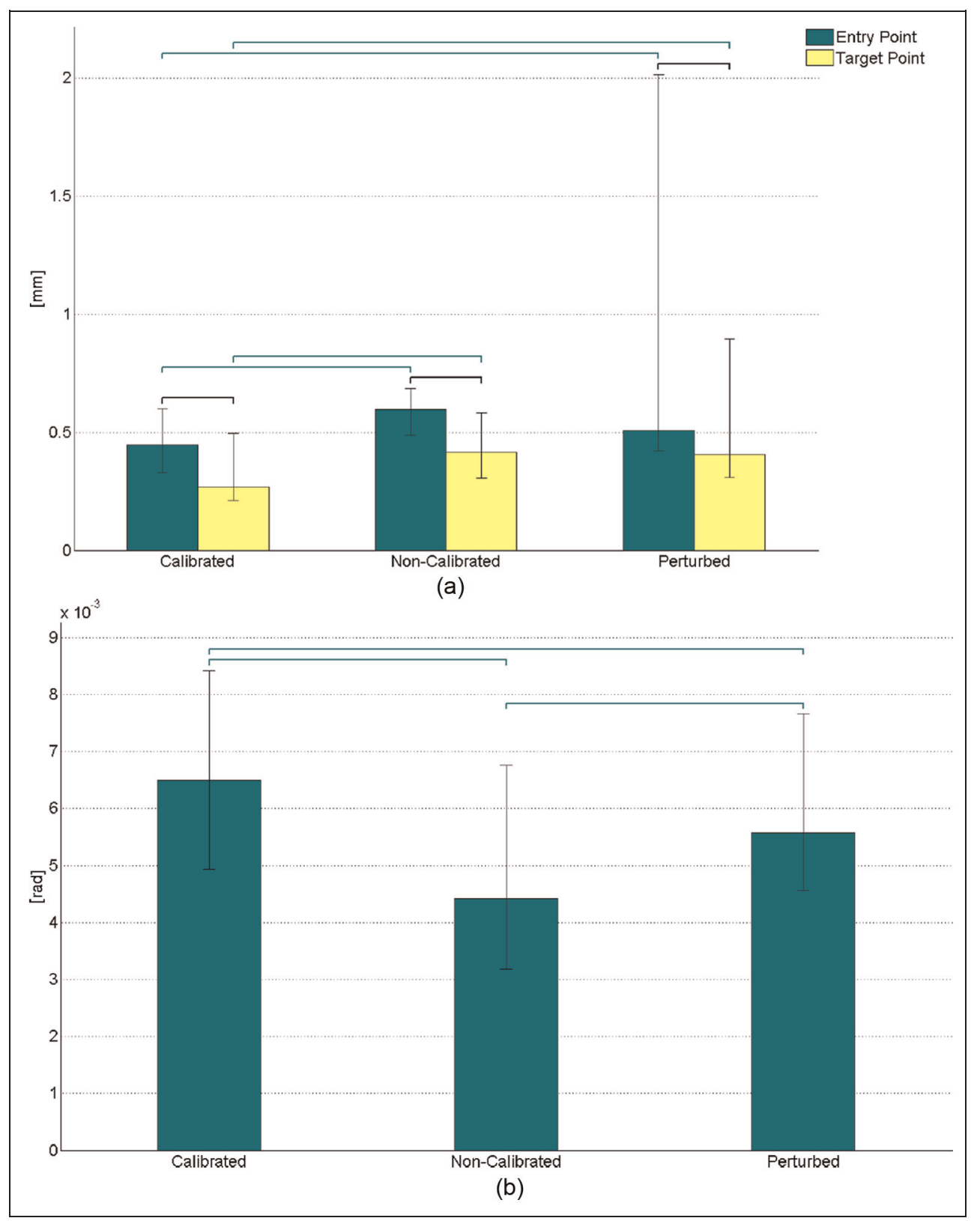

Figure 9. Errors between planned trajectory and real one. Bars represent the median value and the error bars show the interquartile range of the data presented. Horizontal bars indicate significant difference $(p<0.05)$. (a) Targeting distances at the entry point $R E^{E P}$, at the target point $R^{T P}$ in the three evaluation scenarios (calibrated, non-calibrated and perturbed). (b) Angular error $A^{E P}$ among the planned and the actual probe trajectory in the three evaluation scenarios (calibrated, non-calibrated and perturbed).

different from the targeting error at the target point ( $0.4 \mathrm{~mm}$ as a median value), and both values satisfy the requirements for clinical applications $(1 \mathrm{~mm}$ is the typical stereotactic frame accuracy). Fiducial registration error $^{33}$ (which was estimated to be about $0.1 \mathrm{~mm}$ as a median value) influences the results, but with an amplitude of the same order as the optical localization error.

The tracking system, which provides overall surveillance further to registration and targeting corrections, also increases the safety of the application since the consistency of the calibration loop, together with the visibility of all DRFs, is continuously checked at $10 \mathrm{~Hz}$ by a safety check (similar to that proposed by Baron et al. ${ }^{17}$ ). Whenever an inconsistency is detected, probe advancement into the brain is stopped and the system placed in a safe state.

In conclusion, the objective of the work was reached as the targeting accuracy obtained is better than previous work and better than the one required by neurosurgical procedures. The presence of the tracking system is not a further constraint in the OR, since it is usually used for neuronavigation, but in this application it has two further functions: error reduction and safety. 
As already introduced, the targeting algorithm proposed can be easily integrated into previous commercially available surgical robots for keyhole surgery.

\section{Funding}

This work was supported by the EU [grant number ROBOCAST FP7-ICT-215190]

\section{Conflict of interest}

The authors declare that there are no conflict of interests.

\section{References}

1. Joskowicz L, Shamir R, Freiman M, et al. Image-guided system with miniature robot for precise positioning and targeting in keyhole neurosurgery. Comput Aided Surg 2006; 11(4): 181-193.

2. Dogangil G, Davies BL and Rodriguez y Baena F. A review of medical robotics for minimally invasive soft tissue surgery. Proc IMechE, Part H: J Engineering in Medicine 2010; 224: 653-679.

3. Haidegger T, Kovacs L, Fordos G, et al. Future trends in robotic beurosurgery. In: Proceedings of 14th NordicBaltic Conference on Biomedical Engineering and Medical Physics, vol. 20 of IFMBE Proceedings, pp. 229-233. Berlin: Springer, 2008.

4. Cossu M, Lo Russo G, Francione S, et al. Epilepsy surgery in children: results and predictors of outcome on seizures. Epilepsia 2008; 49(1): 65-72.

5. Sutherland GR, Latour I and Greer A. Integrating an image-guided robot with intraoperative MRI: a review of the design and construction of NeuroArm. IEEE Eng Med Biol Mag 2008; 27(3): 59.

6. Ferrand-Sorbets S, Taussig D, Fohlen M, et al. Frameless stereotactic robot-guided placement of depth electrodes for stereo-electroencephalography in the presurgical evaluation of children with drug-resistant focal epilepsy. In: CNS Annual Meeting, 2010.

7. Shamir R, Freiman M, Joskowicz L, et al. Robotassisted image-guided targeting for minimally invasive neurosurgery: planning, registration, and in-vitro experiment. In: Medical Image Computing and ComputerAssisted Intervention-MICCAI, 2005, pp. 131-138.

8. Eljamel MS. Validation of the PathFinder ${ }^{\mathrm{TM}}$ neurosurgical robot using a phantom. Int J Med Robot Comput Assist Surg 2007; 3(4): 372-377.

9. Varma T and Eldridge P. Use of the NeuroMate stereotactic robot in a frameless mode for functional neurosurgery. Int J Med Robot Comput Assist Surg 2006; 2(2): 107-113.

10. Li QH, Zamorano L, Pandya A, et al. The application accuracy of the Neuro-Mate robot - a quantitative comparison with frameless and frame-based surgical localization systems. Comput Aided Surg 2002; 7(2): 90-98.

11. Castillo Cruces RA, Christian Schneider H and Wahrburg J. Cooperative robotic system to support surgical interventions. In: Medical robotics. I-Tech Education and Publishing, 2008.

12. Xia T, Baird C, Jallo G, et al. An integrated system for planning, navigation and robotic assistance for skull base surgery. Int J Med Robot Comput Assist Surg 2008; 4(4): 321-330.

13. Burgner J, Raczkowsky J and Wörn H. End-effector calibration and registration procedure for robot assisted laser material processing: tailored to the particular needs of short pulsed $\mathrm{CO}_{2}$ laser bone ablation. In: Proceedings of 2009 IEEE International Conference on Robotics and Automation, 2009, pp. 2136-2141. IEEE Press.

14. Strobl KH and Hirzinger G. Optimal hand-eye calibration. In: Proceedings of the 2006 IEEE/RSJ International Conference on Intelligent Robots and Systems (IROS), Beijing, 2006, pp. 4647-4653.

15. Cornellà J, Elle OJ, Ali W, et al. Improving Cartesian position accuracy of a telesurgical robot. In: IEEE International Symposium on Industrial Electronics (ISIE), 2008, pp. 1261-1266.

16. Cornellà $\mathrm{J}$, Elle $\mathrm{OJ}$, Ali W, et al. Intraoperative navigation of an optically tracked surgical robot. In: Medical Image Computing and Computer-Assisted Intervention MICCAI 2008 (eds D. Metaxas, L. Axel, G. Fichtinger and G. Szekely G), PT II, vol. 5242 of Lecture notes in Computer Science, 2008, pp. 587-594.

17. Baron S, Eilers H, Munske B, et al. Percutaneous innerear access via an image-guided industrial robot system. Proc IMechE, Part H: J Engineering in Medicine 2010; 224: 632-648.

18. Tobergte A, Fröhlich FA, Pomarlan M, et al. Towards accurate motion compensation in surgical robotics. In: Proceedings of 2010 IEEE International Conference on Robotics and Automation (ICRA), Anchorage, 2010, pp. 4566-4572. IEEE.

19. Zimmermann M, Krishnan R, Raabe A, et al. Robotassisted navigated endoscopic ventriculostomy: implementation of a new technology and first clinical results. Acta Neurochir 2004; 146(7): 697-704.

20. De Momi E and Ferrigno G. Robotic and artificial intelligence for keyhole neurosurgery: the ROBOCAST project, a multi-modal autonomous path planner. Proc IMechE, Part H: J Engineering in Medicine 2010; 224: 715-727.

21. Comparetti MD, Vaccarella A, De Lorenzo D, et al. Multi-robotic approach for keyhole neurosurgery: the ROBOCAST project. In: Proceedings of 2011 Joint Workshop on New technologies for Computer/Robot Assisted Surgery, Graz, Austria, 2011, pp. 1-4.

22. Comparetti MD, De Momi E, Vaccarella A, et al. Optically tracked multi-robot system for keyhole neurosurgery. In: Proceedings of 2011 IEEE International Conference on Robotics and Automation (ICRA), Shangai, 2011, pp. 661-666.

23. Deacon G, Harwood A, Holdback J, et al. The Pathfinder image-guided surgical robot. Proc IMechE, Part H: J Engineering in Medicine 2010; 224: 691-713.

24. De Lorenzo D, Manganelli R, Dyagilev I, et al. Miniaturized rigid probe driver with haptic loop control for neurosurgical interventions. In: Proceedings of $20103 r d$ IEEE RAS \& EMBS International Conference on Biomedical Robotics and Biomechatronics, Tokyo, Japan, 2010, pp. 522-527.

25. Vaccarella A, Cerveri P, De Momi E, et al. A new IGSTK-based architecture for the integration of multimodal sensors and robots in neurosurgical robotics applications. In: Proceedings of the 24th International Congress and Exhibition, 2010, vol. 5, pp. 308-309. 
26. Vaccarella A, Enquobahrie A, Ferrigno G, et al. Modular multiple sensors information management for computer integrated surgery. Int J Med Robot Comput Assist Surg. Epub ahead of print 8 March 2012. DOI: 10.1002/rcs.1412.

27. De Momi E, Cerveri P, Gambaretto E, et al. Robotic alignment of femoral cutting mask during total knee arthroplasty. Int J Comput Assist Radiol Surg 2008; 3(5): 413-419.

28. Jolliffe I. Principal component analysis. In: Encyclopedia of statistics in behavioral science. London, John Wiley \& Sons, 2005.

29. Kumar S, Premkumar P, Dutta A, et al. Visual motor control of a 7DoF redundant manipulator using redundancy preserving learning network. Robotica 2009; 28: 1-16.

30. Chen SJS, Hellier P, Gauvrit JY, et al. An anthropomorphic polyvinyl alcohol triple-modality brain phantom based on Colin27. IEEE Trans Med Imaging 2010; 13(2): 92-100.

31. Surry K, Austin H, Fenster A, et al. Poly(vinyl alcohol) cryogel phantoms for use in ultrasound and MR imaging. Phys Med Biol 2004; 49(24): 5529-5546.

32. Besl PJ and McKay ND. A method for registration of 3-D shapes. IEEE Trans Pattern Anal Machine Intell, 1992, pp. 239-256.

33. West JB and Maurer CR. Designing optically tracked instruments for image-guided surgery. IEEE Trans Med Imaging 2004; 23(5): 533-545.

34. Morgan PS, Carter T, Davis S, et al. The application accuracy of the Pathfinder neurosurgical robot. Int $J$ Comput Assist Radiol Surg 2003; 1256: pp. 561-567.

\section{Appendix I}

\section{Notation}

$\begin{array}{ll}d & \text { desired } \\ f & \text { final }\end{array}$

$i$

j

B

C

M

\section{$\mathbf{R}$}

$\mathbf{T}$

$\mathbf{X}$

Y iterator for the robot in the kinematic

chain

iteration index for the iterative algorithm

robot pose

robot correction transformation

transformation between the tool and the

robot end effector

residual transformation

tool geometrical transformation

calibration matrix for the robot end

effector

calibration matrix for the robot base

\section{Abbreviations}

DoF degree of freedom

DRF dynamic reference frame

EP entry point

FP fine positioner

GP gross positioner

IC iterative correction

LA linear actuator

MRI magnetic resonance image

OR operating room

PI proportional integral

PKM parallel kinematic machine

PT probe trajectory

RC rotation component

TC translation component

TP target point 